\title{
Virtualisierungen der Umwelt: Augmented Reality
}

Eine der wichtigsten aktuellen Formen, in der die Lebensumwelt virtualisiert erscheint, ist die sogenannte Augmented Reality (AR): „This paper surveys the field of Augmented Reality, in which 3-D virtual objects are integrated into a 3-D real environment in real time.“ (Azuma 1997, 355). Augmented Reality bedeutet also: In Echtzeit werden digital generierte Informationen auf die Ansichten realer Objekte vor Ort überlagert. ${ }^{1}$ In Abschnitt 1) wird die AR historisch von der ,Virtual Reality“ (VR) differenziert. In 2) werden einige Anwendungen der AR dargestellt und problematisiert. In 3) folgt ein knappes Fazit.

\section{AR und VR}

Am besten kann die AR dadurch konturiert werden, indem der Unterschied zur VR herausgestellt wird. ${ }^{2}$ Der Grundgedanke der VR war, eine immersive, ggf. durch entsprechende Display- und Interaktionstechniken der*die Benutzer ${ }^{\star}$ in mehr oder weniger umschließende, simulierte Umgebung $\mathrm{zu}$ schaffen, in der jene ${ }^{\star}$ r Benutzer ${ }^{\star}$ in nichts mehr von der ihn* sie eigentlich umgebenden Außenwelt mitbekommt. ${ }^{3}$ Demgegenüber ist die Idee der AR, Elemente simulierter mit Elementen realer Umgebung zu verbinden. Dadurch soll die Realitätswahrnehmung ,verbessert' (augmented) werden, z. B. indem bestimmte Arten von bildlicher, schriftlicher oder akustischer Information auf das Bild des Realraums überlagert werden - eine Überlagerung, die sich idealer an die sich je verändernde Wahl des Wahrnehmungsausschnitts des gegebenen Ortes anpasst und daher immer in Echtzeit sein muss. Insofern es also darum geht, audio-visuell

1 Vgl. zu einigen der informatischen Hintergründe: Bimber und Raskar 2005 und Haller et al. 2007. Die kultur- und medienwissenschaftliche Debatte zu AR ist klein, vgl. Fahle 2006: Fahle bezieht sich wesentlich auf ein spezielles AR-Projekt an der Bauhaus Universität Weimar und dessen bildtheoretische Implikationen. Vgl. auch Manovich 2006: Manovich wiederum behandelt AR nur als Untermenge seiner Beschäftigung mit dem ,augmented space‘ und erwähnt die hier diskutierte Nutzung von Smartphones eher am Rande.

2 Vgl. Milgram et al. 1994 zur Einordnung von AR und VR auf ein Kontinuum verschiedener ,Mixed Realities'.

3 Vgl. zum Folgenden detaillierter Schröter 2004a, auf 166-168 finden sich Anmerkungen zur Genealogie des Begriffs des ,Virtuellen', die für den vorliegenden Aufsatz maßgeblich sind.

○ Open Access. (C) 2021 Jens Schröter, publiziert von De Gruyter. (cc))BY-NC-ND Dieses Werk ist lizensiert unter einer Creative Commons Namensnennung - Nicht-kommerziell - Keine Bearbeitung 4.0 International Lizenz. 
dargestellte Informationen mit dem gerade gegebenen Umraum am gerade gegebenen Ort zu verbinden, sind AR-Applikationen geradezu prototypische Beispiele für orts- und situationsbezogene Medienprozesse.

Im Folgenden seien einige knappe Hinweise zur Archäologie der AR gegeben, die einerseits zeigen, dass das Konzept (aus guten Gründen) schon am Anfang jener Entwicklung angelegt war, die aber andererseits am Ende der 1980er Jahre zunächst in den Diskurs zur VR mündete. Einer der Namen, die immer wieder genannt werden, wenn es um die Geschichte von VR geht, ist Ivan Sutherland (vgl. Schröter 2007). Das liegt erstens daran, dass er 1966 seinen Aufsatz „The Ultimate Display“ veröffentlichte, in dem er eine ultimative Visualisierungstechnologie visionierte, deren Bilder ununterscheidbar von der Realität wären - man sieht mithin, woher die Szenarien z. B. von The Matrix (Wachowski 1999) kommen (vgl. Sutherland 2007). Sutherland beschreibt sozusagen die finale Bildumwelt. Auch in der Theoriebildung der 1990er Jahre wurden diese Vorstellungen perpetuiert, so schrieb Elena Esposito noch 1995: „In einem vollendend [sic] gelungenen Projekt virtueller Wirklichkeit soll der Realitätseffekt so wirkungsvoll sein, dass die Objekte nicht mehr von den Objekten der von der Maschine unabhängigen ,realen Wirklichkeit unterschieden werden können.“ (187). Aber Sutherland war nicht nur der erste ,Visionär der VR. Zweitens und wichtiger hat er wirkliche technische Entwicklungen zu der Genealogie von AR wie VR beigesteuert - insbesondere das am Anfang der 1990er Jahre als ,Datenbrille' geradezu zur Ikone der VR aufgestiegene Head Mounted Display (HMD). Abb. 1 zeigt ein typisches Bild der Zeit.

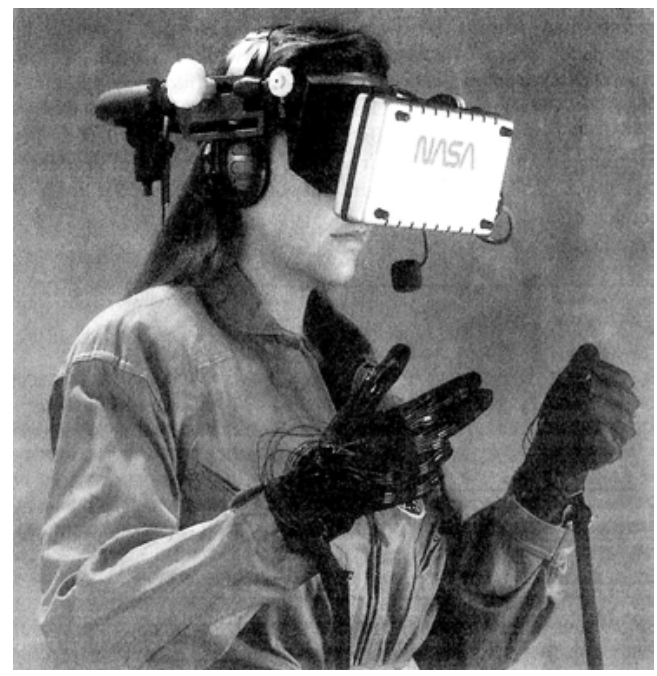

Abb. 1: ,Datenbrille‘ als typische Darstellung von VR, ca. Anfang der 1990er Jahre. 
Sutherland und seine Mitarbeiter entwickeln bis 1968 das erste HMD. Ihre Arbeit wird 1969 in einem Aufsatz mit dem Titel A Head-mounted Three Dimensional Display publiziert. Die ersten Abschnitte umreißen die Grundidee:

The fundamental idea behind the three-dimensional display is to present the user with a perspective image which changes as he moves. [. . .] The image presented by the threedimensional display must change in exactly the way that the image of a real object would change for similar motions of the user's head. [. . .] Our objective in this project has been to surround the user with displayed three-dimensional information.

(Sutherland 1968, 757)

Zunächst klingt das alles nach VR: Die ${ }^{\star}$ der Benutzer^in wird von Information environmental ,umgeben“ und die ständige Neuberechnung des Bildes - abhängig von der Bewegung der ${ }^{\star}$ des Nutzers ${ }^{\star}$ Nutzerin - führt dazu, dass sich die virtuelle Umgebung für die Wahrnehmung auf dieselbe Weise verändert wie es beim Anblick realer Objekte der Fall wäre. (Natürlich ging es zu der Zeit dieses Artikels um einfache Wireframe-Grafiken.) Doch was bei der Einordnung dieses ersten Textes in die Genealogie der VR manchmal übersehen wird, ist, dass Sutherlands HMD halbdurchlässig war und so die Überlagerung der ComputerBilder mit den Bildern des Realraums erlaubte:

Half-silvered mirrors in the prisms through which the user looks allow him to see both the images from the cathode ray tubes and objects in the room simultaneously. Thus displayed material can be made either to hang disembodied in space or to coincide with maps, desk tops, walls, or the keys of a typewriter.

(Sutherland 1968, 759)

D. h. Sutherland hatte bei der Entwicklung des HMD gar nicht das Ziel, einen (den`die Betrachter`in abschottenden) immersiven Raum zu schaffen. Das HMD war als ein Interface konzipiert, welches die sinnfällige und komplexitätsreduzierte Präsentation von Information (z. B. für die wissenschaftliche Visualisierung oder militärische Zwecke - siehe die „maps“, die Sutherland nennt) ermöglichen sollte. HMDs sollten eher zur Effizienzsteigerung des Subjektes dienen. ${ }^{4}$ In diesem Sinne ist er gerade kein Vorläufer der illusionistisch-eskapistisch gedachten VR der frühen neunziger Jahre. Für diesen Diskurs ein Beispiel:

Jaron Lanier wird oft als Erfinder des Begriffs Virtual Reality dargestellt und galt lange als der VR-Guru (vgl. Hayward 1993, 198-200). Er stellte mit seiner Firma VPL auch die ersten kommerziell erhältlichen VR-Systeme (Markennamen: EyePhone und DataGlove) her. In Laniers Auffassung wird die virtuelle Umgebung trotz des sonst immer beschworenen Realismus' keineswegs von

4 Vgl. z. B. HMDs als spezielle Displays für Kampfpiloten: Furness 1986. 
vorneherein auf eine realistische Wiedergabe realer Szenerien und realer Körper festgelegt. Wozu auch? Eine VR zu schaffen, die die äußere Wirklichkeit einfach verdoppelt, ist sinnlos. Also fordert Lanier die Fiktionalisierung der VR. Es stehe, so Lanier, ein ganzes Spektrum von Möglichkeiten zur Verfügung, welches auch die Selbstrepräsentation des`der Users`in als fiktionale Figur einräumt. In der VR, so führt Lanier aus, „könnte [man] ohne weiteres ein Gebirge sein oder eine Galaxie oder ein Kieselstein auf dem Boden“ (1991, 72). So wird, jedenfalls im Prinzip, auch eine freie Fiktionalisierung des eigenen Körpers möglich - auch wenn unklar bleibt, was es genau heißen soll, eine ,Galaxie zu sein'. Lanier unterstreicht mehrfach den widerspenstigen Charakter der materiellen und körperlichen Welt: „Das Tragische an der physischen Wirklichkeit ist, dass sie zwingend ist“ $(1991,81)$.

Laniers Diskurs zeigt ziemlich deutlich, was die Attraktion der VR als andere Welt, in die man gleichsam flüchten zu können glaubte, ausmachte. Es schien möglich, das Gefängnis der physischen Realität zu verlassen. Vielleicht ist es kein Zufall, dass solche Vorstellungen um 1990 florierten. 1989/90 endete der Kalte Krieg, das ,Ende der Utopien“ wurde verkündet - und so stießen utopische Aufladungen der neuen Computertechnologien in jenes Vakuum. Diese utopischen Aufladungen sind genau das, was heute in den Science and TechnologyStudies, bei Jasanoff oder bei Kirby, als sozio-technisches Imaginäres bezeichnet wird (vgl. Kirby 2010; Jasanoff und Kim 2015). So bemerkte Bernhard Waldenfels: „Es könnte sein, daß die ,alteuropäischen' Geschichtsillusionen nach ihrem Niedergang abgelöst werden durch technologische Allmachtsphantasien aus der Neuen Welt.“ (1998, 197). Die in der VR angeblich mögliche Befreiung vom eigenen Körper führt Lanier zu der den utopischen Stellenwert verdeutlichenden These, dass die VR ,die absolute Aufhebung von Klassen- und Rassenunterschieden und allen anderen vorgeschobenen Formen [bedeutet], da alle Formen veränderlich sind.“ (1991, 83). Auch dies ist nach 1989 lesbar als eine verschobene Wiederkehr der ansonsten obsolet gewordenen Sozialutopien, die gerade die Überwindung von sozialer Ungerechtigkeit und Rassismus versprochen hatten.

Es dürfte kaum noch überraschen, dass sich VR (zumindest in dieser starken Form) nie etabliert hat, auch wenn sich gegenwärtig mit Techniken wie der Oculus Rift ein gewisses Comeback der VR anzubahnen scheint. So ist die Erzeugung eines auch nur einigermaßen überzeugend wirkenden virtuellen BildTon-Raums technisch schwierig, die Simulation der Tasterfahrung (etwa durch ,Datenhandschuhe') ist umständlich und aufwendig, die VR-Wahrnehmung stößt auf Probleme wie den Konflikt zwischen audiovisueller und propriozeptiver Wahrnehmung (,Simulatorkrankheit‘) und kollektive Rezeptionsprozesse sind behindert. Vor allem aber ist ihre eskapistische Funktion kaum mit den Funktionalitätsimperativen der globalen kapitalistischen Weltordnung nach 
1989/90 vereinbar (wie das auch für Drogen gilt). Wenn VR-ähnliche environments heute genutzt werden, dann in Simulatoren, um Subjekte zu trainieren und für spezifische Aufgaben zu optimieren. Dabei geht es gerade nicht darum, die Welt durch eine VR zu ersetzen, sondern vielmehr, die Welt mithilfe virtueller Räume zu beherrschen und zu kontrollieren. Unsere hochtechnische HochrisikoKultur (Flugzeuge, Atomkraftwerke etc.) benötigt solche „control environments“ (Ellis 1991, 327), um überhaupt operieren zu können (vgl. Schröter 2004b).

Doch dass die schon bei Sutherland angelegte Möglichkeit der AR, also einer Überlagerung des virtuellen Raums mit dem realen Ort, heute - von den Trainingssimulatoren abgesehen - immer wichtiger wird, verwundert nicht. Während die VR (zumindest in ihrer phantasmatischen Form) die Flucht aus dieser Welt erlauben soll, dient die AR dazu, sie mit Informationen anzureichern, d.h. zu funktionalisieren und zu optimieren. Daher ist sie heute viel wichtiger - und ihre Durchsetzung letztlich ein Zeichen dafür, dass neue Medien in der Regel nicht (oder nicht nur) die Welt fundamental verändern, sondern in die dominanten Strukturen integriert werden, um sie z. B. zu beschleunigen und dadurch z. B. Produktivitätsvorteile in der kapitalistischen Konkurrenz zu erzeugen (Was nicht heißt, dass die neuen technischen Verfahren nicht auch zu Verschiebungen, Störungen und Konflikten führen.).

\section{2}

Insbesondere der Ausbreitung der Smartphones ist es zu verdanken, dass wir jetzt alle die Welt in Echtzeit mit Daten und Informationen überlagern können. Einen schönen Überblick über mehr als 50 AR-Apps für das iPhone liefert die Website Iphoness mit dem Artikel 50 + Best Augmented Reality iPhone Applications (Ci 2019).

Bei diesen zukunftsweisenden Anwendungen wird die Kamera des iPhones genutzt, um das Bild des Ortes auf dem Display sichtbar zu machen und in Echtzeit mit Informationen zu überlagern. Man kann an dieser Website grob und heuristisch drei verschiedene Kategorien von AR-Anwendungen unterscheiden: 1. Orts-optimierende Applikationen (Informationsanreicherung); 2. Orts-ludische Applikationen (Spiel) und 3. Orts-ästhetische Applikationen (Gestaltung). Im Folgenden sollen diese verschiedenen Formen und ihre Implikationen diskutiert werden. 


\subsection{Orts-optimierende Applikationen}

Wie Abb. 2 zeigt, sind viele der Apps für die Zulieferung geographischer Information gedacht. Es geht darum, sich in einer gegebenen Umgebung besser orientieren zu können, indem GPS-Daten, das Bild eines Kompasses etc., dem Ort in Echtzeit überlagert werden. Ganz praktische Anwendungen können dazugehören: So kann man den Ort, an dem man sein Auto geparkt hat, taggen, um so leicht zum Auto zurückzufinden (Wobei für eine solche Funktion eigentlich keine Bildüberlagerung notwendig ist.). D. h. der Raum wird funktionalisiert, um Zeitersparnis zu erzielen. Damit kann die AR dazu dienen, die Figur des

\section{AR navigation apps for iOS}

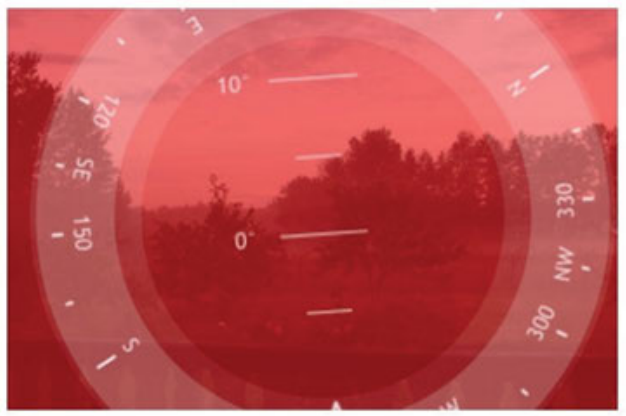

SpyGlass: turns your iPhone into a spyglass. Shows you a compass, GPS info, and much more on the go. Very useful for outdoors.

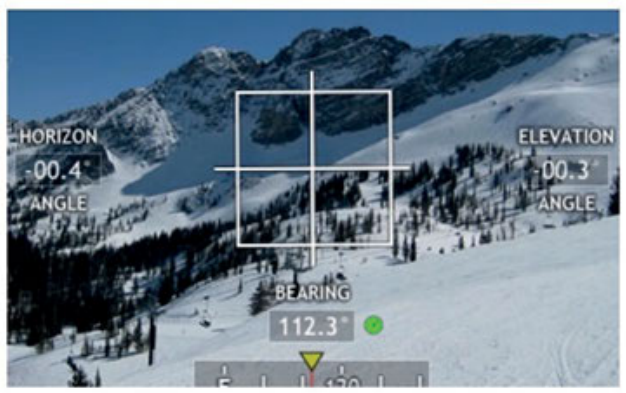

Theodolite: a cool little app that acts as your compass, GPS, map, and two-axis inclinometer. Great for hiking and active sports in general.

Abb. 2: Ausschnitt des Artikels 50 + Best Augmented Reality iPhone Applications: Orts-optimierende Applikationen. 
Flaneurs, wie sie sich in der Moderne in der Literatur von Baudelaire, Benjamin u. a. herausgebildet hat, auszustreichen. Wenn die „Minimaldefinition“ gilt, „daß der Flaneur richtungs- und ziellos durch die Großstadt streift“ (Neumeyer 1999, 17), ${ }^{5}$ dann kann er mit einer ,Poetik des Nichts-Tuns“ in Verbindung gebracht werden. ${ }^{6}$ Und insofern das ,richtungs- und ziellose Herumstreifen` auch eine Verweigerung von Effizienz und Funktionalität ist, kann AR als Technologie der Effizienzsteigerung des Subjekts verstanden werden. ${ }^{7}$ Außerdem bedeutet die Möglichkeit der Orientierung notwendig zugleich, dass die Position der Nutzer ${ }^{\star}$ in bekannt sein muss und - wie Skandale darum gezeigt haben - auch von den Smartphones gespeichert werden kann: „The close connection between surveillance/monitoring and assistance/augmentation is one of the key characteristics of the high-tech society. “ (Manovich 2006, 222). Die Optimierung des sich bewegenden Subjekts ist also eine doppelte: Nicht nur wird die Bewegung selbst effizient gemacht, sondern es fallen potentiell auch Bewegungsprofile an, die weniger einer politischen Überwachung als einer kommerziellen Verwertung zugeführt werden dürften.

Zugleich können Hintergrund-Informationen aus Datenbanken wie z.B. Wikipedia mit dem Bild überlagert werden; es geht also darum, die Umgebung mit Bedeutung aufzuladen. So heißt es in dem Artikel $50+$ Best Augmented Reality iPhone Applications zu Wikitude: „[A]nother cool augmented iPhone application that helps you explore your surroundings effectively on your phone“ (Ci 2019). So praktisch das ist, es bleibt durchaus die Frage, wie man diese Operationalisierung der Umgebung durch ihre Überlagerung mit virtuellen Informationsräumen einstufen soll. Ein Paper mit dem schönen Titel 7 Things You Should Know About Augmented Reality (Educause Learning Initiative 2005) diskutiert den didaktischen Einsatz von AR und spricht dabei ganz offen aus, dass eine der Möglichkeiten der AR ist, das Lernen auf den Alltag auszudehnen, gewissermaßen alles in Weiterbildung zu verwandeln. Hierin kann man ein Element der von Deleuze beschriebenen kontrollgesellschaftlichen Ordnung sehen, in der „die permanente Weiterbildung tendenziell die Schule [. . .] und die kontinuierliche Kontrolle das Examen“ (Deleuze 1990, 257) ablöst. Ähnlich, wie dank der geomedialen Apps z. B. keine Zeit bei der Suche nach dem Auto verloren werden soll, wird die Freizeit selbst zum Weiterbildungsraum: Beide Male geht es darum, Subjekte und ihr

5 Vgl. ein schönes Beispiel bei Bergman: „Wir streiften absichtslos durch die Stadt, verliefen uns, fanden uns wieder zurecht, verliefen uns erneut.“ $(1987,197)$.

6 Vgl. Fuest 2008, insbesondere Kapitel III.

7 Im Grunde kann die Archäologie der Effizienzsteigerung durch ,Augmentierung“ bis zu Douglas Engelbarts Programm einer ,Augmentation of Human Intellect‘ durch den gezielten Einsatz von Computern zurückverfolgt werden; vgl. Engelbart 1962. 
Handeln zu optimieren. Dabei kann zumindest die Frage aufgeworfen werden, ob die Überlagerung der Dinge durch Informationen nicht auch den Interpretationsspielraum einschränkt und zu einer Homogenisierung der Dingerfahrung beiträgt. Durch Ausbreitung von AR-Apps auf Smartphones könnte sich also auch eine homogenisierte Interpretation der Dinge ausbreiten - gleichsam als globale semantische Matrix, die Teil der Globalisierungsprozesse ist.

\subsection{Orts-ludische Applikationen}

Ein großer Teil der Apps in dem Artikel $50+$ Best Augmented Reality iPhone Applications sind Spiele.

Dabei wird das Bild des Ortes in Echtzeit mit Spielfiguren oder Ähnlichem überlagert, in einer genannten Applikation kann man durch das iPhone auf seine Füße sehen und es wird das Bild eines Fußballs überlagert, die App erkennt die Füße und man kann den virtuellen Ball vor sich her kicken (Abb. 3). Solche Casual Games sind ideale Werkzeuge des Zeitvertreibs, z. B. beim Warten oder auf dem Weg zur Arbeit und tragen als solche zur Operationalisierung der immer stärker geforderten Mobilität bei.

Bei Spielen geht es offenkundig nicht darum, wie in den in 2.1) genannten Anwendungen, die außerhalb befindliche Realität mit Informationen anzureichern, um sie zu funktionalisieren, sondern darum, den (meist) bekannten Umraum zum Setting der Spiele selbst zu machen. So ersetzt nicht ein virtuelles Spiel-Bild den Blick auf die Außenwelt, vielmehr kann diese neu erfahrbar gemacht werden. Ein geradezu kindliches Vergnügen an der Neuentdeckung der Welt wird eröffnet. Ein gewisser Harald Ebert von Nintendo bemerkt genau in diesem Sinn: „Da wird der eigene Wohnzimmertisch zum Videospiel-Level.“ (Ebert 2011) Zugleich erfordert der Umgang mit den AR-Spielen unter Umständen auch eine unablässige Bewegung des Körpers, um die Konsole zu bewegen, damit immer neue Abschnitte des Realraums sichtbar und überlagert werden.

Die steigende Beliebtheit von AR-Spielen - oder vielmehr AR-,Spielereien' scheint Ausdruck einer stetigen (demographischen) Erweiterung der Computerspiel-Kultur zu sein: Ihren vorläufigen Höhepunkt dürfte dies mit Pokemon Go erreicht haben. So ist zu beobachten, dass Computerspiele immer breitere Bevölkerungsschichten ansprechen und der Stereotyp der ,Hardcore-Gamer`in “ mittlerweile eher die Ausnahme denn die Regel bildet (vgl. Newman 2004). Gerade der Siegeszug der sogenannten Casual Games - Jesper Juul spricht zurecht von einer „Casual Revolution“ (vgl. Juul 2010) - markiert hier einen wichtigen Entwicklungsschritt, der nicht zuletzt auch vom Erfolg der Nintendo Wii (mit ihren neuen Interface-Möglichkeiten) mitgetragen wurde. Wenn z. B. mehr unterwegs 


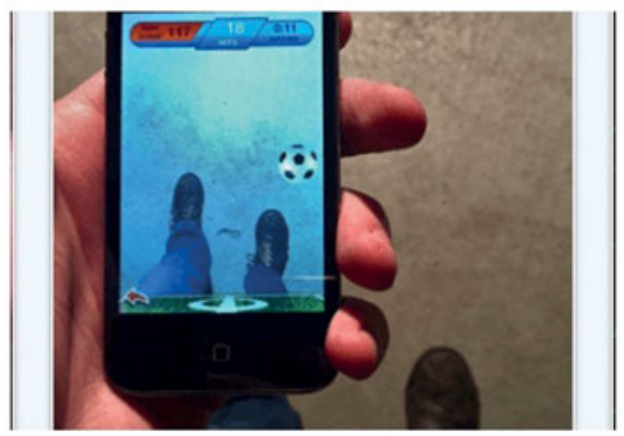

AR Soccer: as suggested by a few of our readers, this is soccer meets augmented reality. Gives you a virtual ball to play with at all times.

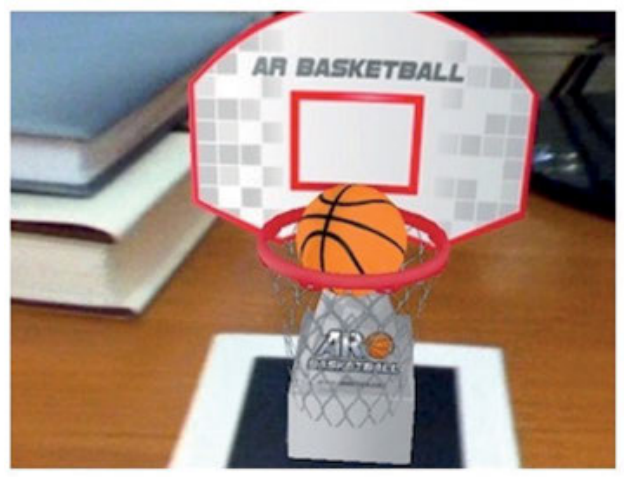

AR Sports Basketball: this ARKit app lets you play an augmented reality game of basketball on your desk.

Abb. 3: Ausschnitt der in dem Artikel 50+ Best Augmented Reality iPhone Applications gezeigten Orts-ludischen Applikationen.

gespielt wird, liegt der Reiz des AR-Spielens gerade darin, den jeweiligen Ort, an dem man sich befindet, spielerisch zu nutzen. Es bleibt abzuwarten, ob Nintendo auch mit dem 3DS neue Spielergruppen zu erschließen vermag. ${ }^{8}$ Der interessante Kontrast der hier diskutierten Anwendungen $\mathrm{zu}$ 2.1) ist jedenfalls, dass es nicht um eine Optimierung, sondern um eine Ludisierung des Orts in Echtzeit geht.

8 Mit Dank an Benjamin Beil. 


\subsection{Orts-ästhetische Applikationen}

Schließlich wird auf der genannten Website über die besten AR-Apps für das iPhone eine App genannt, die nicht recht zu den bisherigen beiden Kategorien passen will (Abb. 4).

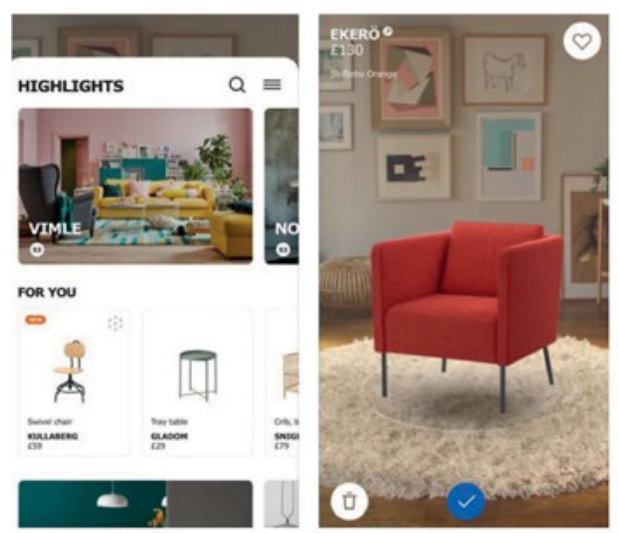

IKEA Place: this iPhone augmented reality app uses ARKit to let you try IKEA items at your place.

Abb. 4: Ausschnitt des Artikels 50 + Best Augmented Reality iPhone Applications: Orts-ästhetische Applikation.

Bei diesem Programm namens Ikea Place des schwedischen Möbelhauses Ikea geht es darum, die Bilder des Ortes in Echtzeit mit Abbildungen von Möbelstücken zu überlagern und in diesem Sinne gestaltend auf die eigene Umgebung zuzugreifen. Daher wurde der Begriff der ,Orts-ästhetischen Applikation“ vorgeschlagen, selbst wenn es sich nicht im engeren Sinne um eine künstlerische Anwendung handelt - obwohl es solche natürlich gibt (s. u.).

Die AR-Anwendung reduziert also die Ängste (und erneut: den Zeitverbrauch), die mit dem Möbeleinkauf einhergehen können, insofern sie vorab zu testen erlaubt, ob sich ein Möbelstück in die heimische Umgebung einfügen wird. Sieht man einmal von der sich so anzeigenden Schwäche visueller Vorstellungskraft ab, ist erneut eine optimierende Funktion der AR-Applikation unübersehbar. Sie funktioniert gewissermaßen als neue Form des Katalogs, die die im Katalog isoliert präsentierten Waren zu situieren erlaubt und so eine bessere Abschätzung ermöglicht, ob das evtl. zu erwerbende Objekt sich in die übergreifende Gestaltung des eigenen Wohnraums einfügt. Der Katalog beginnt 
den Realraum zu überlagern. Zunächst soll also der Warenkauf erleichtert werden. Offenkundig implizieren derartige Anwendungen eine Ästhetisierung, da Fragen nach dem Preis, der Verarbeitung etc. eines Möbelstücks zugunsten der Frage danach zurücktreten, ob das Objekt im Umraum der eigenen Wohnung ästhetisch ,funktioniert‘. Insofern zeigt sich hier auch eine - im Sinne Bourdieus - soziale Segmentierung, die nicht ganz überraschend ist. Nutzer^innen, die sich ein iPhone leisten können, können meistens auch die Frage nach den Kosten eines Möbelstücks zugunsten ihrer Selbststilisierung zurückstellen. Zugleich ist diese Selbststilisierung, z. B. durch eine ,stimmige‘ Einrichtung, eine Option des Differenzgewinns bzw. der ,Individualisierung‘ gegenüber anderen. Insofern ist diese AR-Applikation eine Selbsttechnologie (Foucault) für die ästhetizistische Individualitätsproduktion postmoderner Konsumenten. Die Differenzproduktion ist für die Markt- und Markendiversifizierung essentiell, weil für die Konsument*innen der Anschluss an bestimmte Gestaltungsweisen als ,Selbstsein' erscheint und so gerade den Eindruck einer Heteronomie durch eine ,Kulturindustrie“ (Adorno/Horkheimer) umgehen kann - was übrigens auch für Apple selbst gelten dürfte. Insofern AR-Applikationen den Ort in Echtzeit zum permanenten Gestaltungsfeld dieser schein-autonomen Differenzpraxis machen, sind sie eine Herrschaftstechnologie.

Aber es gibt auch andere ästhetisierende Praktiken. So können AR-Verfahren tatsächlich ein Ansatzpunkt für künstlerische Praktiken sein. Auch für Smartphones gibt es AR-Kunstprojekte.

Diese Bilder dokumentieren ein Projekt, das am 9. Oktober 2010 durchgeführt wurde (Abb. 5 und 6). Besucher`innen mit den entsprechenden Smartphones und AR-Software (Wikipedia contributors 2019) können im MoMA an einer virtuellen und inoffiziellen Ausstellung teilnehmen:

The virtual exhibition will occupy the space inside the MoMA building using Augmented
Reality technology. The show will not be visible to regular visitors of the MoMA, but those
who are using a mobile phone application called „Layar Augmented Reality browser“ on
their iPhone or Android smartphones, will see numerous additional works on each of the
floors.
(Veenhof 2010) D. h. mit AR wird der Raum des MoMAs gewissermaßen besetzt und die autoritative Selektion der Werke und die Narration ihrer Anordnung unterlaufen, durchbrochen und so verschoben. Das kann durchaus als subversiver Angriff auf die hegemoniale Funktion des MoMAs verstanden werden (Allerdings kann die AR-Ausstellung ebenso als Anerkennung der hegemonialen Rolle des MoMA gewertet werden.). Hier deuten sich kritische Potentiale einer AR-Kunst an, die die stabilisierten räumlichen Strukturen neuen Deutungs- und Wahrnehmungsweisen öffnet. 


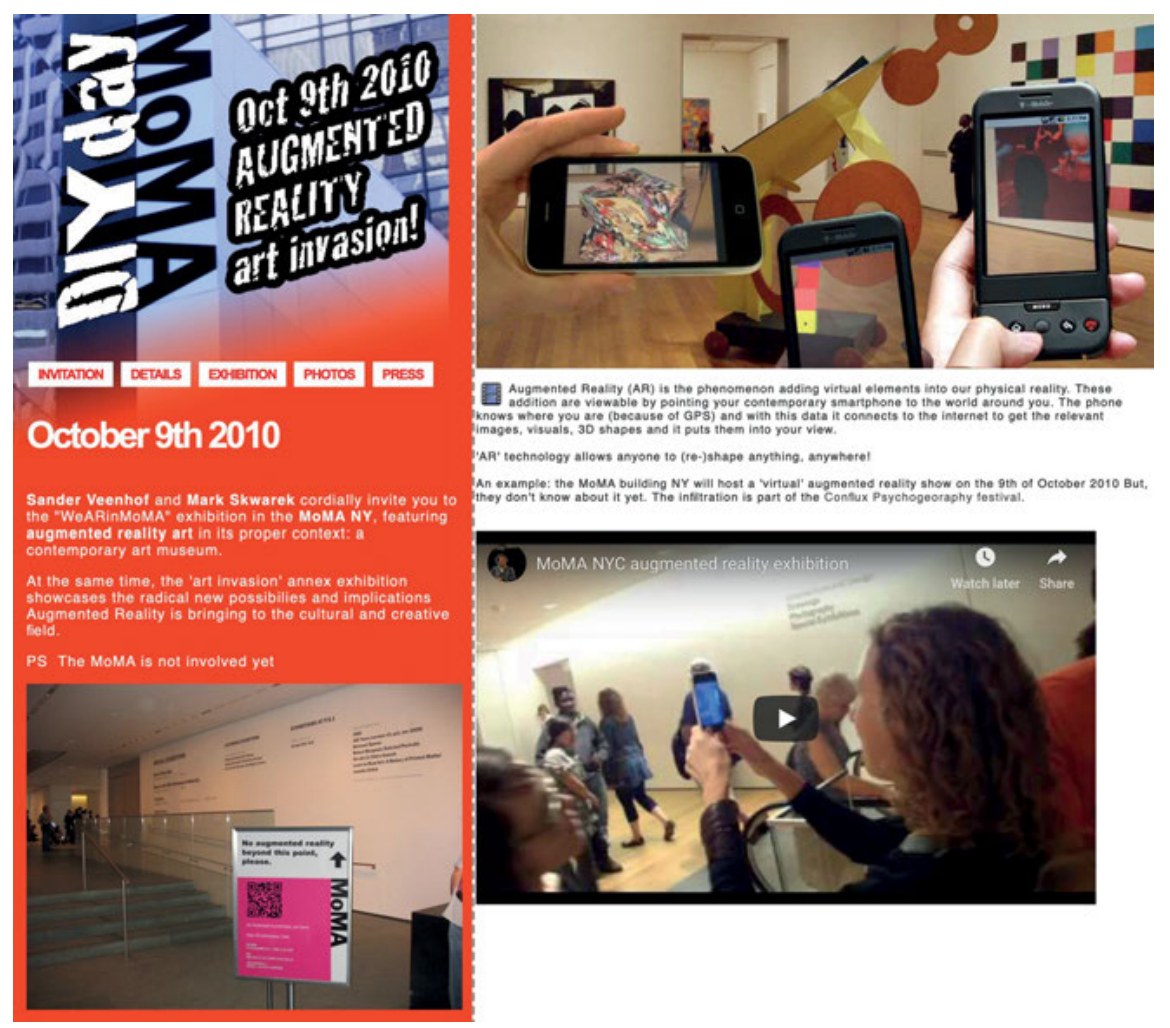

Abb. 5 und 6: Augmented Reality Art Invasion 2010, http://www.sndrv.nl/moma/.

\section{Fazit}

E8s zeigt sich, dass mit der AR ein wichtiger Bereich von Bildumwelten angesprochen ist, welcher eine große Bandbreite von kontrollgesellschaftlichen Optimierungen, Ludisierungen und Ästhetisierungen erlaubt, aber auch kritische Potentiale enthält. Bildumwelten, besonders in Simulatoren oder eben in optimierenden AR-Anwendungen, haben Funktionen der Kontrolle, aber die Vielfalt der Anwendungen lässt sich wohl nicht darauf reduzieren. Die genauen Einsatzformen konkret in ihrer Situierung zu untersuchen - dies ist bei AR per definitionem unumgänglich -, ist eine zentrale Aufgabe der (medienethnographischen) Erforschung situierter Medien. Eine Medienästhetik der AR (oder der AR als eine Form einer generelleren ,Mixed Reality‘ [vgl. Milgram et al. 1994]) hätte systematisch darzulegen, wie auditiv und visuell virtuelle und reale Räume und Objekte aufeinander bezogen werden und welche Parameter dabei ausschlaggebend 

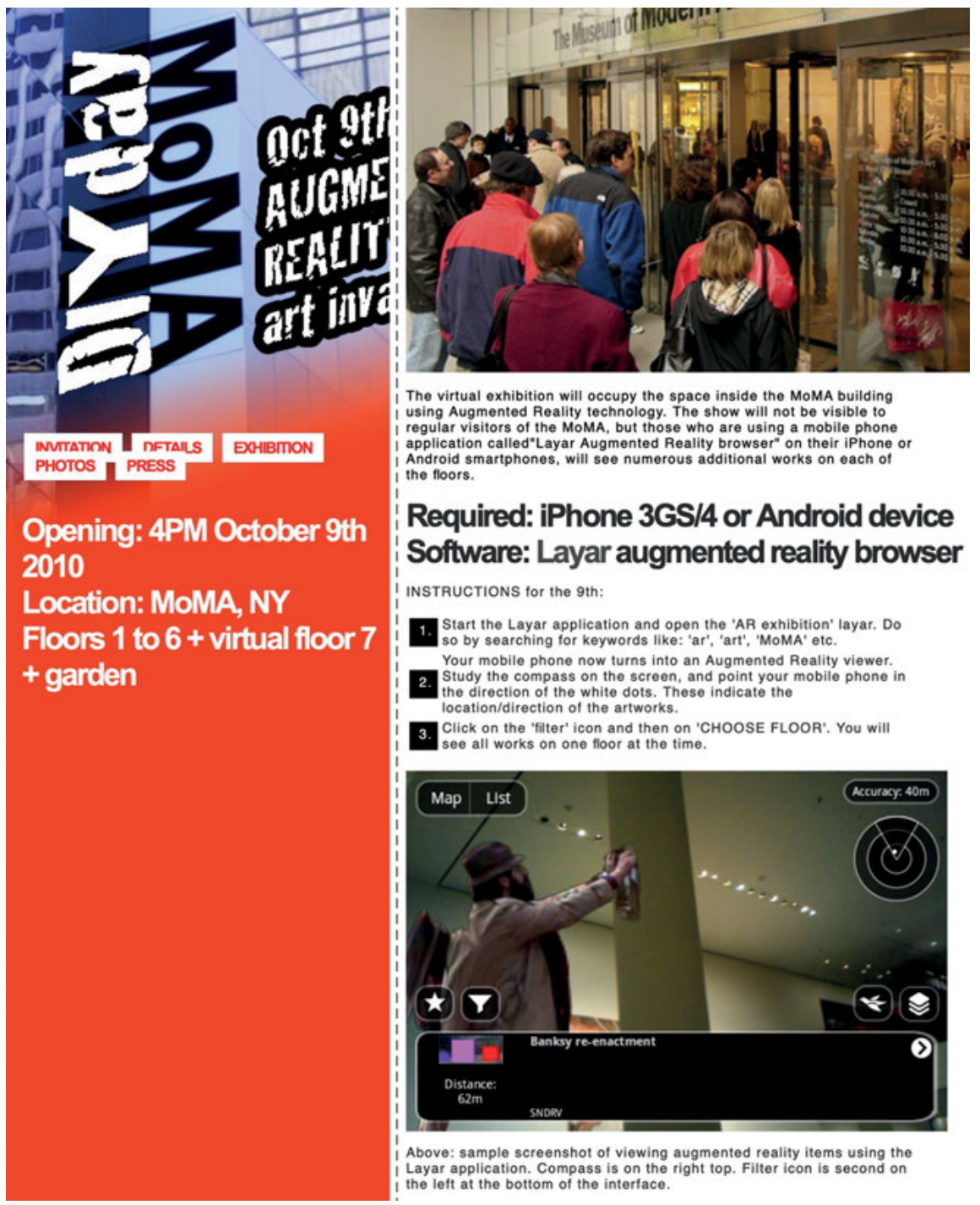
Required: iPhone $3 G S / 4$ or Android device
Software: Layar augmented reality browser INSTRUCTIONS for the 9th:

1. Start the Layar application and open the 'AR exhibition' layar. Do so by searching for keywords like: 'ar', 'art', 'MoMA' etc. Your mobile phone now turns into an Augmented Reality viewer. 2. Study the compass on the screen, and point your mobile phone in the direction of the white dots. These indicate the location/direction of the artworks.

3. Click on the 'filter' icon and then on 'CHOOSE FLOOR'. You will see all works on one floor at the time.

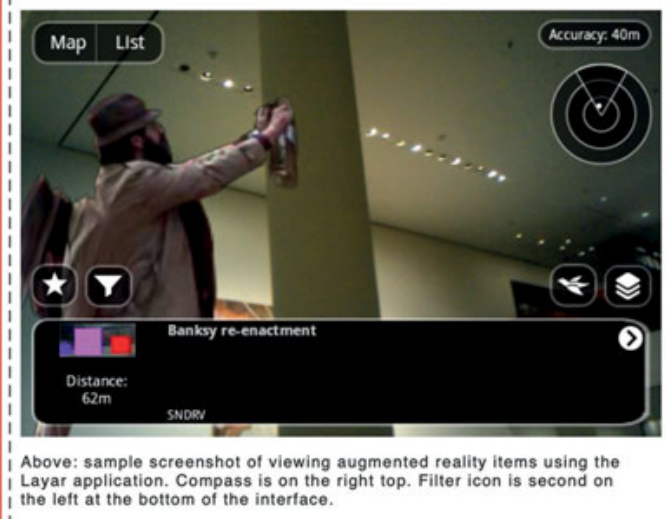

Abb. 5 und 6 (fortgesetzt)

sind (vgl. Schröter 2018). Sie könnte dadurch auch beschreiben, welche Bewegungspfade, Interaktionsweisen und Wahrnehmungspotentiale für die potentiellen Betrachter^innen und Benutzer^innen dabei auf welche Weise ermöglicht oder verstellt werden. Die Frage nach der Differenz von Realem und Virtuellem muss heute zur Frage nach den medienästhetischen Strategien ihrer Verbindung sowie deren politischen Implikationen weiterentwickelt werden. 


\section{Literatur}

Azuma, Ronald T. „A Survey of Augmented Reality“. Presence: Teleoperators and Virtual Environments 6.4 (1997): 355-385.

Bergman, Ingmar. Mein Leben. Hamburg: Hoffmann und Campe Verlag, 1987.

Bimber, Oliver, und Ramesh Raskar. Spatial Augmented Reality. Merging Real and Virtual Worlds. Wellesley, MA: A K Peters, 2005.

Ci. 50+ Best Augmented Reality iPhone Apps. http://www.iphoneness.com/iphone-apps/ best-augmented-reality-iphone-applications/ (29. März 2019).

Deleuze, Gilles. „Postskriptum über die Kontrollgesellschaften“. Unterhandlungen 1972-1990. Frankfurt a.M.: Springer, 1990. 254-262.

Ebert, Harald. Sonstiges: Expertenrunde zum 3DS-Launch. http://www.planet3ds.de/artikel/ 9-expertenrunde-zum-3ds-launch.html. 2011 (29. März 2019).

EDUCAUSE Learning Initiative. 7 Things You Should Know About Augmented Reality. https:// library.educause.edu/resources/2005/10/7-things-you-should-know-about-augmented-re ality. ELI 7 Things You Should Know 2005 (29. März2019).

Ellis, Stephen R. „Nature and Origins of Virtual Environments. A Bibliographical Essay“. Computing Systems in Engineering 2.4 (1991): 321-347.

Engelbart, Douglas C. Augmenting Human Intellect: A Conceptual Framework. http://sloan. stanford.edu/mousesite/EngelbartPapers/B5_F18_ConceptFrameworkInd.html. 1962 (29. März2019).

Esposito, Elena. „Illusion und Virtualität. Kommunikative Veränderungen der Fiktion“. Soziologie und Künstliche Intelligenz. Produkte und Probleme einer Hochtechnologie. Hg. Werner Rammert. Frankfurt a.M., New York: Campus Verlag, 1995. 187-216.

Fahle, Oliver. „Augmented Reality - Das partizipierende Auge“. Das Spiel mit dem Medium. Partizipation - Immersion - Interaktion. Zur Teilhabe an den Medien von Kunst bis Computerspiel. Hg. Britta Neitzel und Rolf F. Nohr. Marburg: Schüren, 2006. 91-103.

Fuest, Leonhard. Poetik des Nicht(s)tuns. Verweigerungsstrategien in der Literatur seit 1800. München: Wilhelm Fink, 2008.

Furness, Thomas. „The Supercockpit and its Human Factors Challenges“. Proceedings of the 30th Annual Meeting of the Human Factors Society (1986): 48-52.

Haller, Michael, Mark Billinghurst und Bruce Thomas. Emerging Technologies of Augmented Reality. Interfaces and Design. Hershey, PA: Idea Group Inc., 2007.

Hayward, Philip. „Situating Cyberspace. The Popularisation of Virtual Reality“. Future Visions. New Technologies of the Screen. Hg. Philip Hayward und Tana Wollen. London: British Film Institute Press, 1993. 180-204.

Jasanoff, Sheila, und Sang-Hyum Kim. Dreamscapes of Modernity. Sociotechnical Imaginaries and the Fabrication of Power. Chicago, London: University of Chicago Press, 2015.

Juul, Jasper. A Casual Revolution. Reinventing Video Games and Their Players. Cambridge, MA: MIT Press, 2010.

Kirby, David. „The Future is Now. Diegetic Prototypes and the Role of Popular Films in Generating Real-world Technological Development“. Social Studies of Science 40.1 (2010): 41-70.

Lanier, Jaron. „Was heißt ,virtuelle Realität? Ein Interview mit Jaron Lanier“. Cyberspace. Ausflüge in virtuelle Wirklichkeiten. Hg. Manfred Waffender. Reinbek bei Hamburg: Rowohlt, 1991. 67-89. 
Manovich, Lev. „The Poetics of Augmented Space“. Visual Communication 5.2 (2006): 219-240.

Milgram, Paul, Haruo Takemura Akira Utsumi und Fumio Kishino. „Augmented Reality: A Class of Displays on the Reality-Virtuality Continuum“. Proceedings of SPIE 2351.1 (1994): 282-292.

Neumeyer, Harald. Der Flaneur. Konzeptionen der Moderne. Würzburg: Könighausen und Neumann, 1999.

Newman, James. Videogames. London, New York: Routledge, 2004.

Schröter, Jens. Das Netz und die Virtuelle Realität. Zur Selbstprogrammierung der Gesellschaft durch die universelle Maschine. Bielefeld: transcript, $2004 \mathrm{a}$.

Schröter, Jens. „Computer/Simulation. Kopie ohne Original oder das Original kontrollierende Kopie“. OriginalKopie - Praktiken des Sekundären. Hg. Gisela Fehrmann, Erika Linz, Eckhard Schumacher und Brigitte Weingart. Köln: DuMont, 2004b. 139-155.

Schröter, Jens. „Von grafischen, multimedialen, ultimativen und operativen Displays. Zur Arbeit Ivan E. Sutherlands“. Display II: Digital. Navigationen. Zeitschrift für Medien- und Kulturwissenschaften 7.2. (2007): 33-48.

Schröter, Jens. „Atmosphäre und Interferenz“. Zur Ästhetik medialer Räume. Mediale Räume. Hg. Stephan Günzel. Berlin: Kadmos, 2018. 111-121.

Sutherland, Ivan. „A Head-mounted Three Dimensional Display“. AFIPS Conference Proceedings 33.1 (1968): 757-764.

Sutherland, Ivan. „Das ultimative Display“. Display II: Digital. Navigationen. Zeitschrift für Medien- und Kulturwissenschaften 7.2 (2007): 29-32 (übersetzt von Nicola Glaubitz und Jens Schröter). The Matrix. Reg. Lana und Lilly Wachowski. Warner Bros, 1999.

Veenhof, Sander. MoMA, Details. http://www.sndrv.nl/moma/?page=details, 2010 (29. März 2019).

Waldenfels, Bernhard. „Ein menschlicher Traum für Wachende. Zur Natürlichkeit und Künstlichkeit der Erfahrung“. Grenzen der Normalisierung. Studien zur Phänomenologie des Fremden 2. Frankfurt a.M.: Suhrkamp, 1998. 196-213.

Wikipedia contributors. Layar. https://en.wikipedia.org/w/index.php?title=Layar\&oldid= 849693898. Wikipedia, The Free Encyclopedia, 10. Juli 2018 (29. März 2019).

\section{Abbildungen}

Abb. 1 ,Datenbrille' als typische Darstellung von VR, ca. Anfang der 1990er Jahre.

Abb. 2 Ausschnitt des Artikels 50+ Best Augmented Reality iPhone Applications: Ortsoptimierende Applikationen.

Abb. 3 Ausschnitt der in dem Artikel 50+ Best Augmented Reality iPhone Applications gezeigten Orts-ludischen Applikationen.

Abb. 4 Ausschnitt des Artikels 50+ Best Augmented Reality iPhone Applications: Ortsästhetische Applikation.

Abb. 5 und 6 Augmented Reality Art Invasion 2010, http://www.sndrv.nl/moma/ (29. März 2019). 
